\title{
Data verification tools for minimizing management costs of dense air-quality monitoring networks
}

Georgia Miskell; Jennifer Salmond; Maryam Alavi-Shoshtari; Mark Bart; Bruce Ainslie; Stuart Grange; Ian G. McKendry; Geoff S. Henshaw; David E. Williams*

\section{Supplementary Material}

\section{Contents}

1. Examples of single sensor assessment of failure

2. The empirical cumulative distribution (ECD) function and Kolmogorov-Smirnov (KS) test formulae

3. Figures;

i. $\quad$ Figure $\mathrm{S} 1$ - map locations of the nine co-located sites

ii. Figure S2 - ozone concentrations over a day for sites in different land-uses

iii. Figure S3 - time-series of the nine co-located GSS instruments

iv. Figure S4 - map locations of the GSS instruments in residential land-uses

v. Figure S5 \& S6 - control charts for the other six co-located instruments

4. Tables;

i. Table $\mathrm{S} 1$ - sensitivity analysis in selecting the time index, $t_{d}$

ii. Table S2 - sensitivity analysis in selecting the time index, $t_{f}$

iii. Table S3 - time of drift for the 18 residential locations 
1. Examples of single sensor assessment of failure: for full details see Bart et al. (2014) and associated Supplementary Information.

a. The low-cost instruments derive a 'zero' by modulating the air flow over the sensor between a low value ('zero' or 'baseline' resistance measurement, $R_{b}$ ) and a high value ('gas' resistance measurement, $R_{g}$ ). The $R_{b}$ value should remain stable over time if the sensor microstructure remains stable. The non-linear relationship between instrument indicated ozone and $\left(R_{g}-R_{b}\right)$ should remain stable if the calibration loaded into the instrument remains valid. Changes in microstructure or air flow rates would change the calibration.
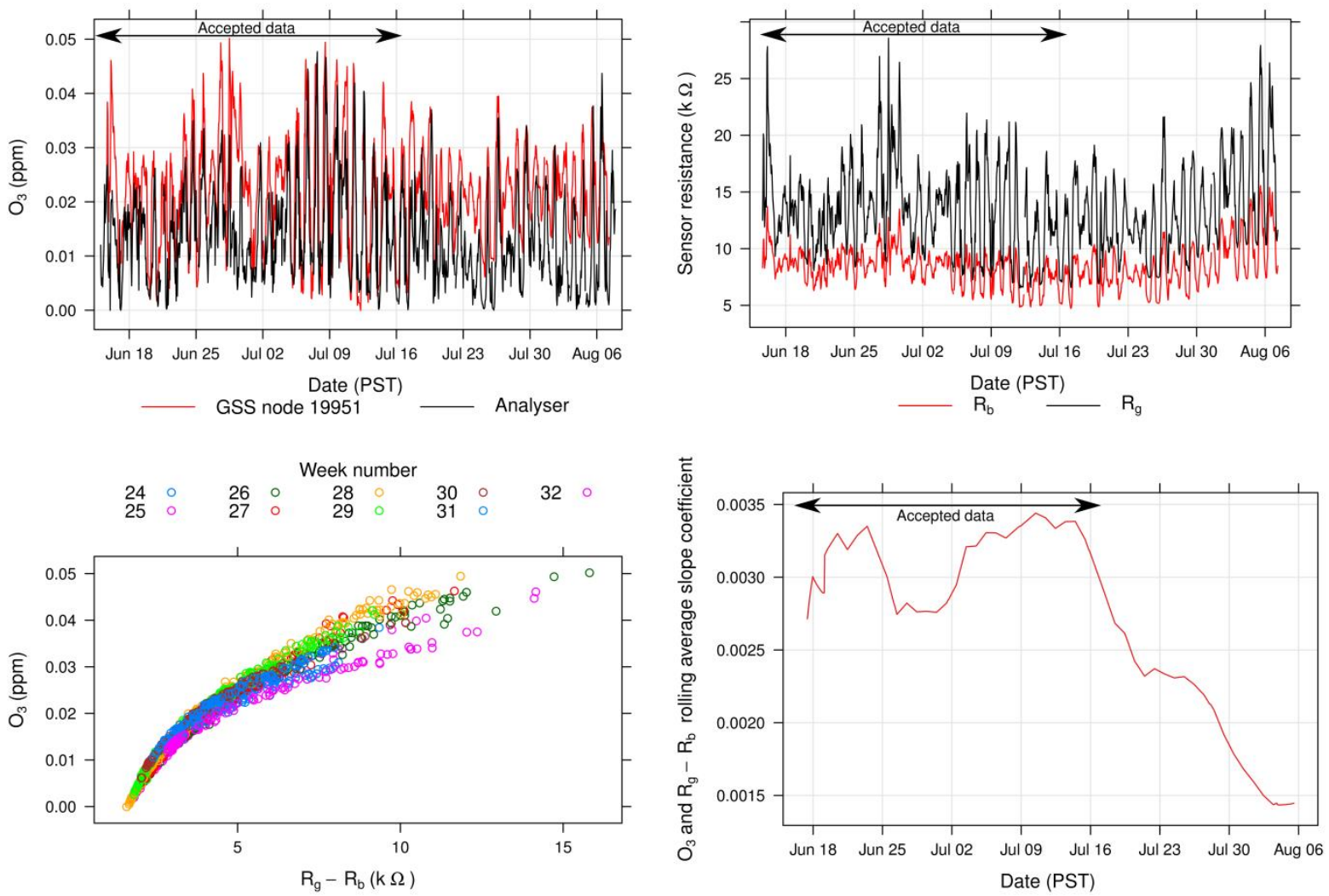

The example shows that at day 211 , a steady increase in $R_{b}$ was discernible which resulted in a systematically higher indicated ozone concentration when ozone was low and that at higher ozone, the value of $R_{g}-R_{b}$ for a given indicated ozone increased. The behaviour was crudely but effectively indicated by average linear correlation slope of indicated ozone vs $\left(R_{g}-R_{b}\right)$ (running slope over a one week $-168 \mathrm{hr}$ - interval). The results are consistent with a change in microstructure of the sensor element. 
b. There is an expectation that that the ozone concentration should usually be low during the night and should show a daily variance that is reasonably consistent over time.

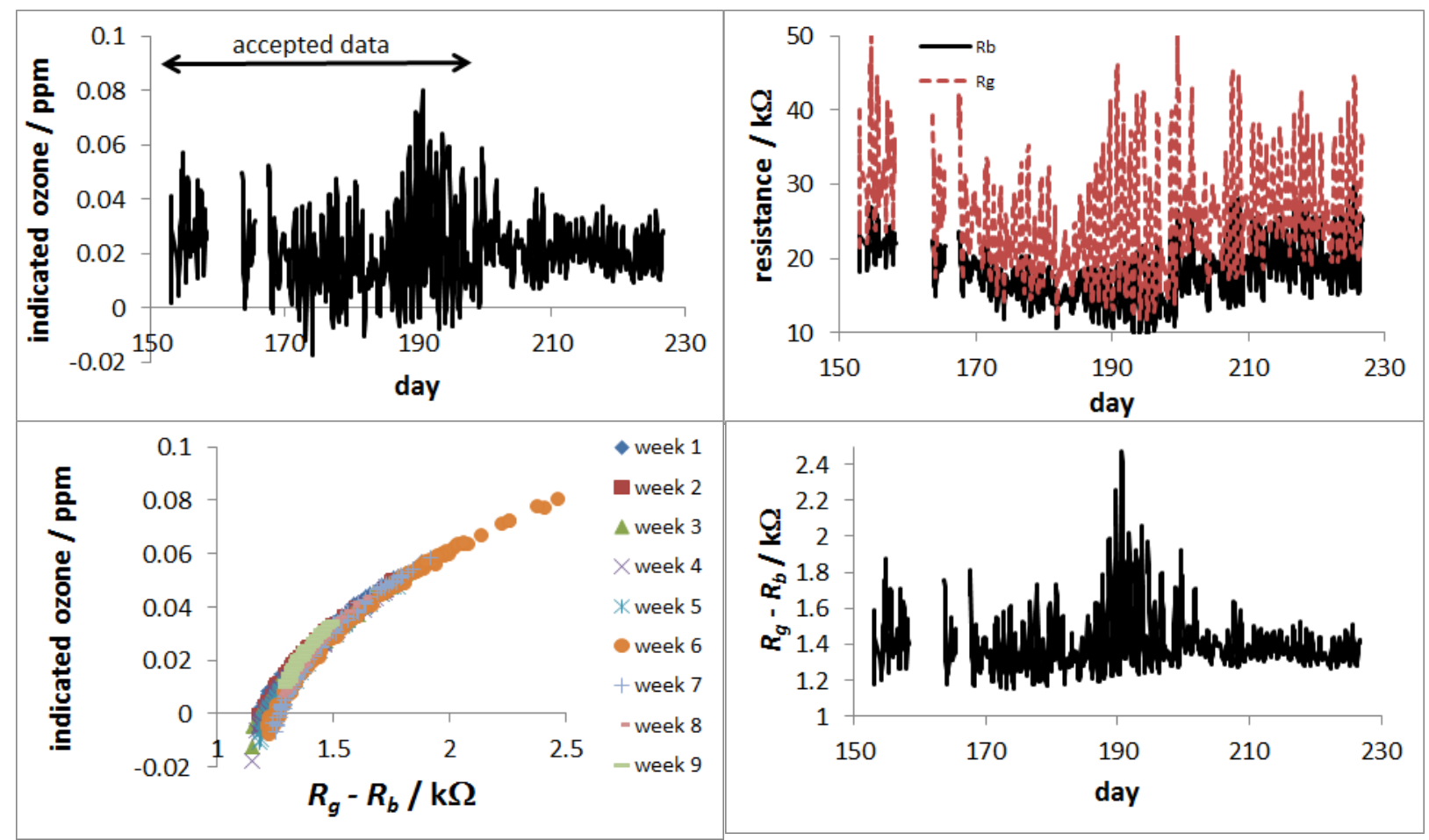

In this example, at day 195, the indicated daily variance of ozone concentration abruptly dropped. $R_{b}$ was relatively stable but the range shown by $R_{g}$ abruptly dropped. The relationship between indicated ozone and $R_{g}-R_{b}$ did not change. Since the device behaviour shows no internal inconsistency, the likely explanation is particle deposition on the sensor causing ozone decomposition within the device. 


\section{The Empirical Cumulative Distribution (ECD) Function \& the Kolmogorov- Smirnov two-sample test:}

The ECD for a sample with $t_{w}$ observations and variable $X$ has the cumulative probability;

$$
\mathbb{P}(x>X)=\hat{F}_{t_{w}}(x)=\frac{1}{t_{w}+1} \sum_{i=1}^{t_{w}} \begin{cases}1, & x>x_{i} \\ 0, & x \leq x_{i}\end{cases}
$$

Assumptions are $x_{i}$ are i.i.d. random variables with a common underlying ECD, $F(X)$.

The K-S statistic uses the ECD over a specified time window, $t_{w}$. The test hypothesises the two samples will have similar distributions by comparing the maximum absolute distance (supremum function, sup) between the cumulative probability curves on the $y$-axis for the same $x$-axis values. The KS statistic, $D$, for two samples with $m, t_{w}$ observations and common variable $X$ is:

$$
D_{m, t_{w}}=\frac{\sup }{x}\left|\hat{F}_{m}(x)-\hat{F}_{t_{w}}(x)\right|
$$

KS statistics can be translated into p-values $\left(p_{K S}\right)$ based on sample size and probability thresholds, with the null hypothesis, $\mathrm{H}_{0}$, being the two samples could come from the same distribution. Assumptions are the two samples are independent, observations are independent, and both come from continuous distributions. For an air quality network the assumptions would be the samples are derived from the same air-shed and over the same time period. 


\section{Figures;}

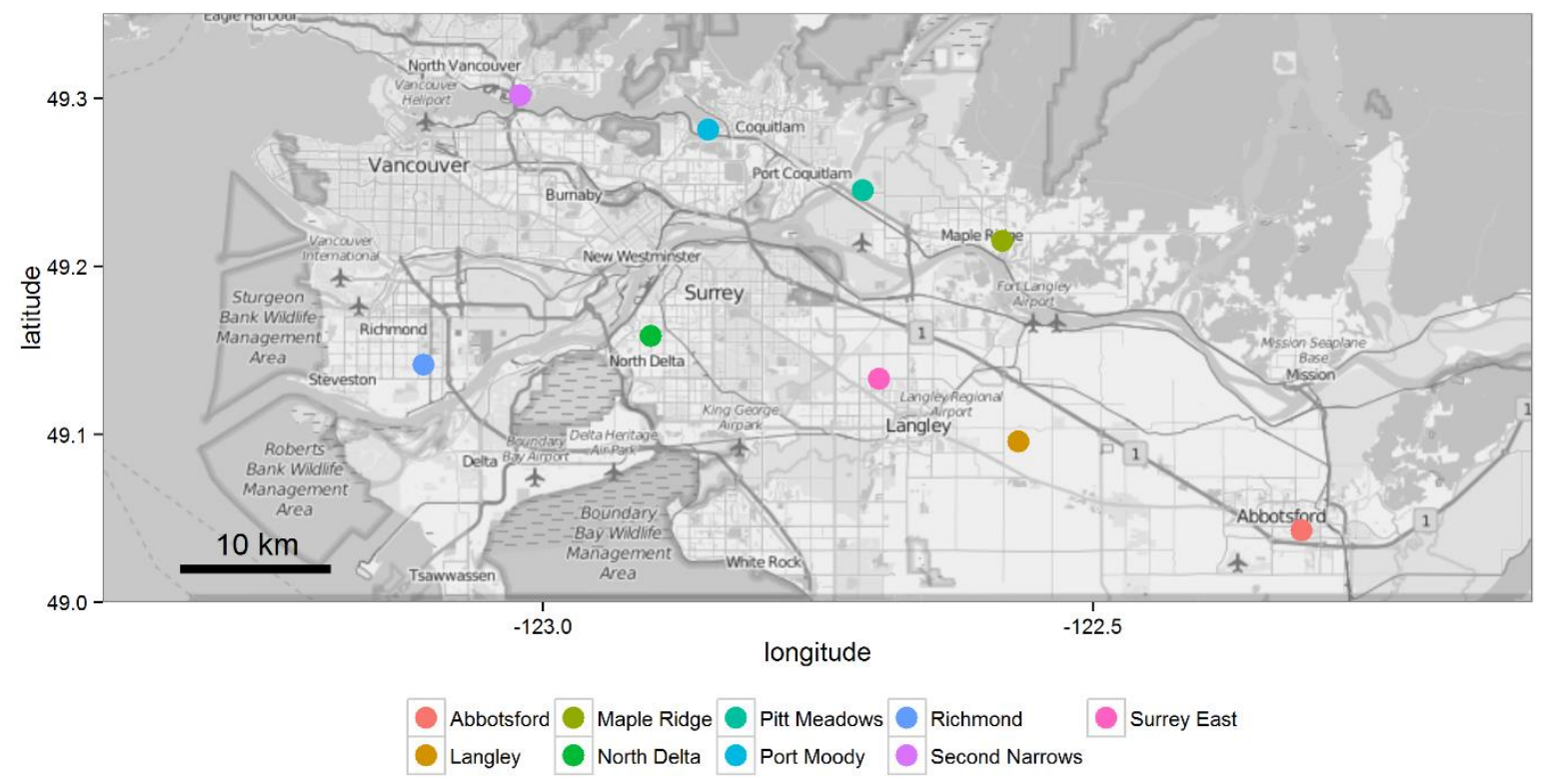

Figure S1: Map locations of the nine sites co-located with reference analysers around the LFV. 


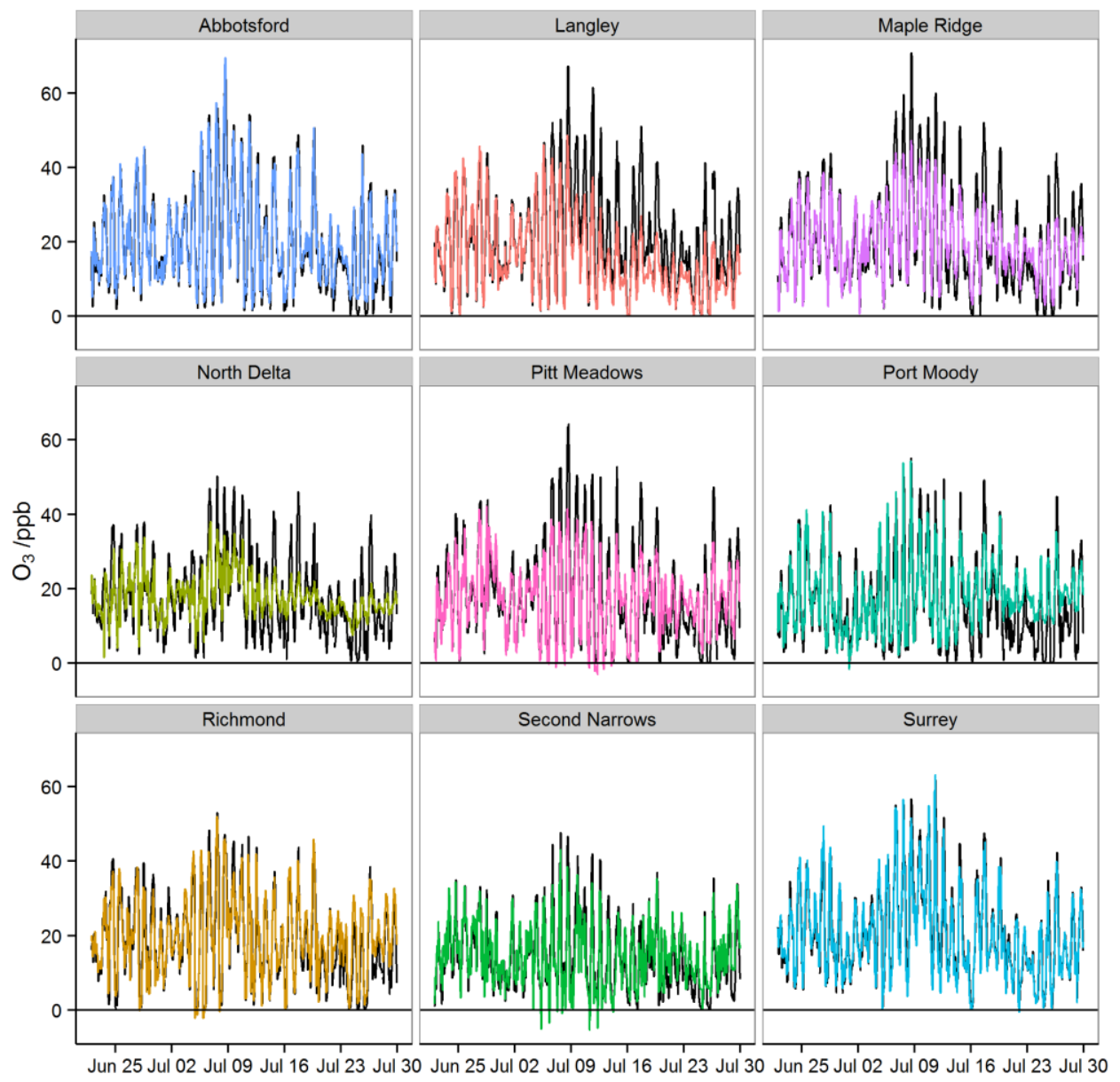

Figure S2: Hourly-averaged field calibrated ozone concentrations from the co-located GSS instruments (in colour) and reference stations (in black). 


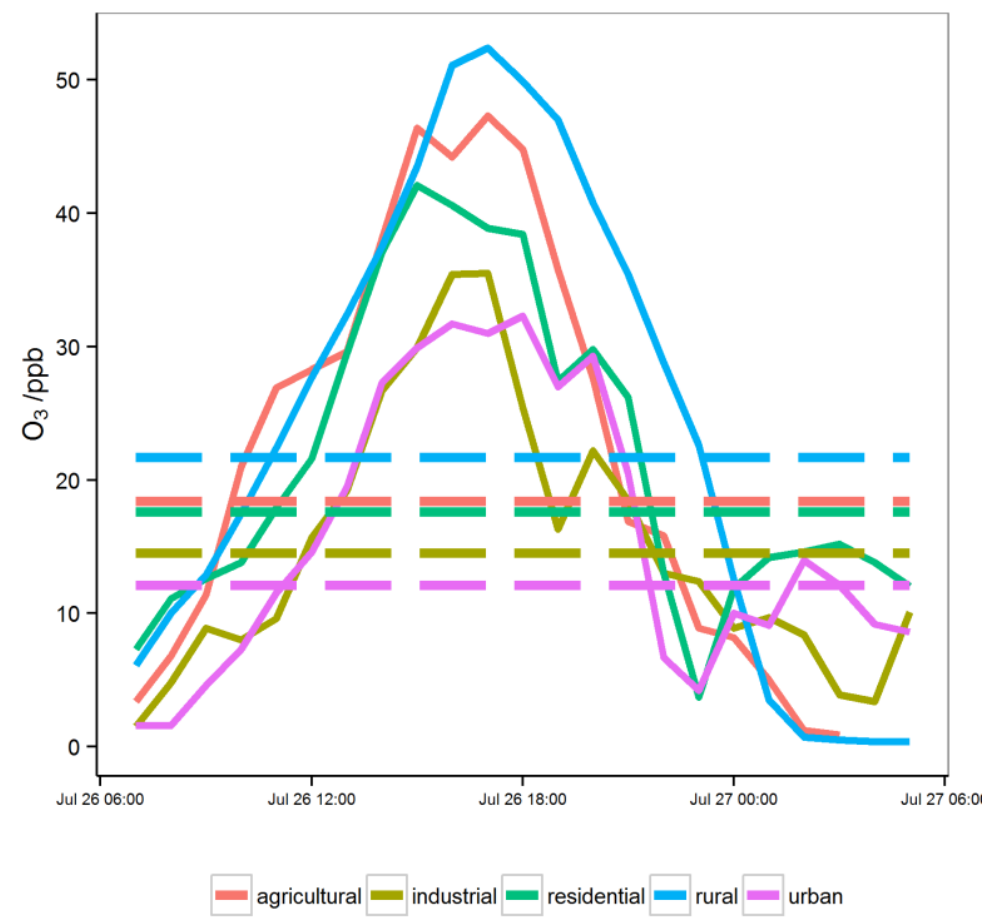

Figure S3: short-term (day, solid lines with diurnal variation) and long-term (three-month average, dashed straight lines) concentrations for reference station sites with different landuses.
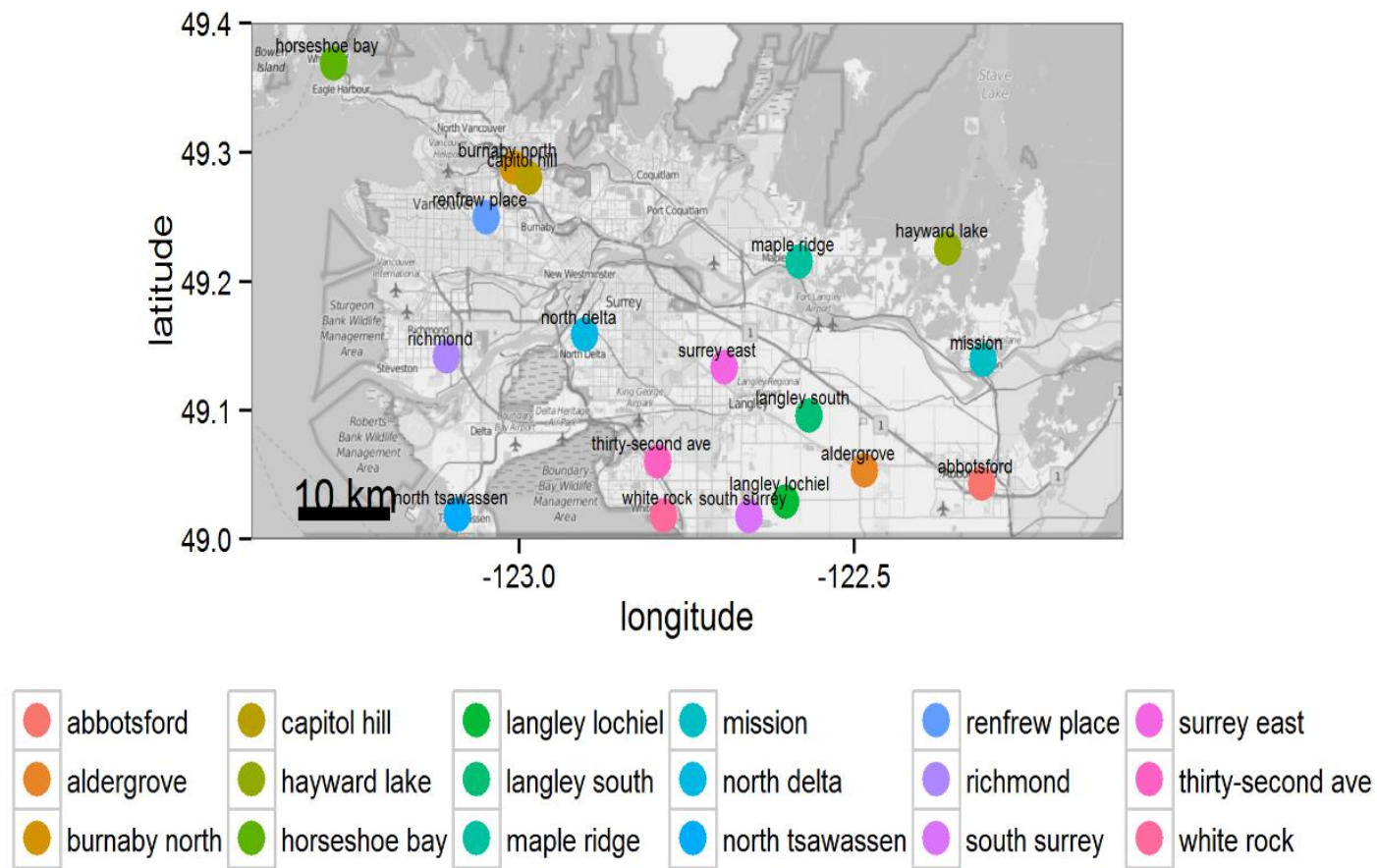

Figure S4: Locations of the 18 low-cost instruments in residential land-uses around the LFV. 

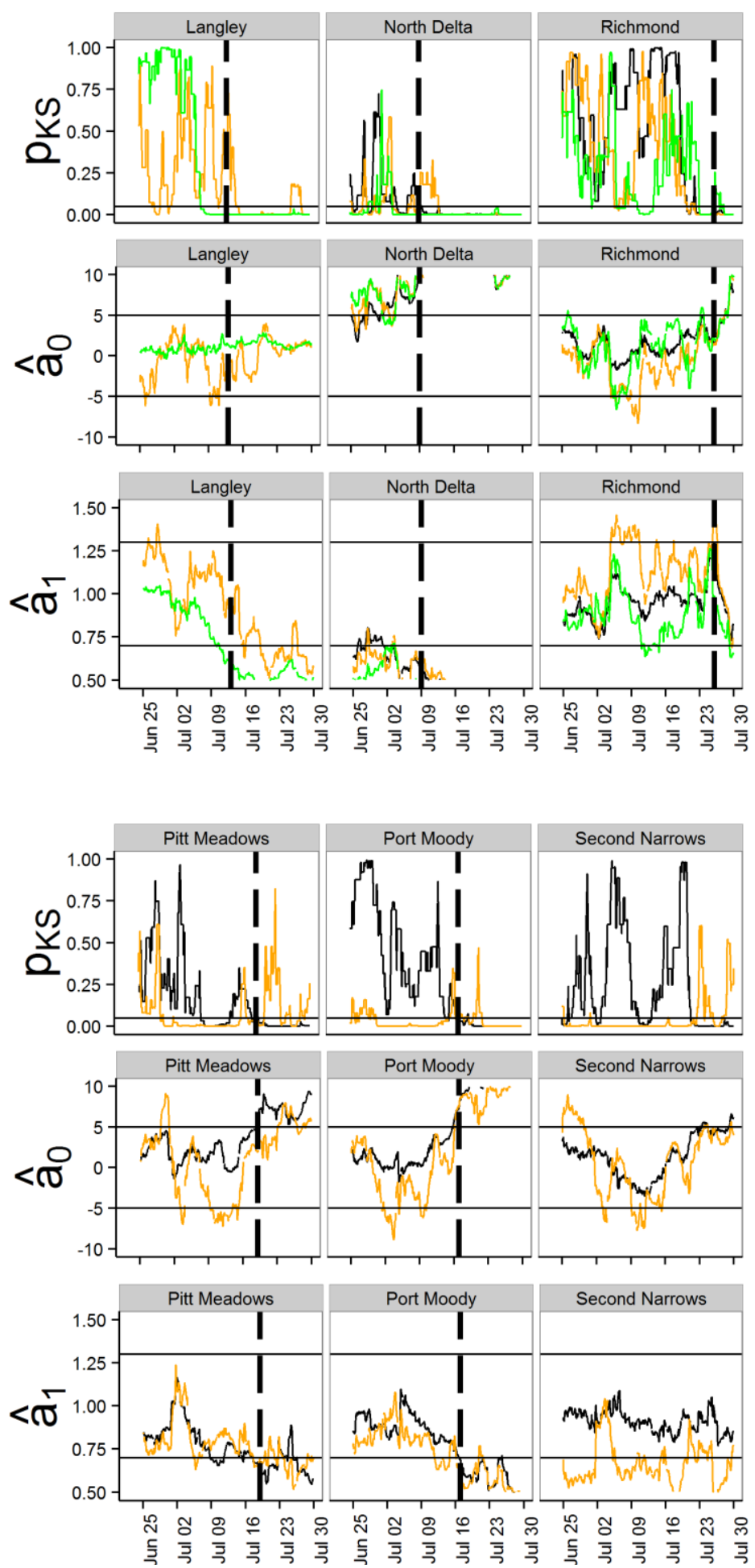

Figure S5 \& S6: Control charts for the other co-located instruments. Black lines are for colocated, green for similar land-use, and orange for different land-use proxies. Langley is the analyser used as the remote residential proxy and so are the same for similar and co-located proxies at this site. S.6 has no similar proxies as these sites were in land-uses that were hard to compare. Dashed vertical lines represent where single sensor assessment identified drift. 


\section{Tables}

Table S1: Sensitivity analysis for the selection of time index, $t_{d}$, using comparisons on Type 1 (False Positive, FP) \& 2 (False Negative, FN) errors based on the co-located GSS instrument data and single sensor assessment (SSA) states $\left(t_{f}=120\right)$ (TP: true positive; TN: true negative)

\begin{tabular}{|c|c|c|c|c|c|c|c|c|c|c|c|c|c|}
\hline \multirow[t]{2}{*}{ Site } & \multirow[t]{2}{*}{ SSA } & \multicolumn{4}{|l|}{$\hat{\mathbf{a}}_{0}$} & \multicolumn{4}{|l|}{$\hat{\mathbf{a}}_{\mathbf{1}}$} & \multicolumn{4}{|l|}{$P_{K S}$} \\
\hline & & $\begin{array}{l}\mathrm{t}_{\mathrm{d}}= \\
24\end{array}$ & $\begin{array}{l}t_{d}= \\
72\end{array}$ & $\begin{array}{l}t_{d}= \\
120\end{array}$ & $\begin{array}{l}t_{d}= \\
168\end{array}$ & $\begin{array}{l}\mathrm{t}_{\mathrm{d}}= \\
24\end{array}$ & $\begin{array}{l}\mathrm{t}_{\mathrm{d}}= \\
72\end{array}$ & $\begin{array}{l}t_{d}= \\
120\end{array}$ & $\begin{array}{l}t_{d}= \\
168\end{array}$ & $\begin{array}{l}t_{d}= \\
24\end{array}$ & $\begin{array}{l}t_{d}= \\
72\end{array}$ & $\begin{array}{l}t_{d}= \\
120\end{array}$ & $\begin{array}{l}t_{d}= \\
168\end{array}$ \\
\hline Abbotsford & OK & $\mathrm{TN}$ & $\mathrm{TF}$ & $\mathrm{TN}$ & $\mathrm{TN}$ & $\mathrm{TN}$ & $\mathrm{TF}$ & $\mathrm{TN}$ & $\mathrm{TN}$ & $\mathrm{TN}$ & $\mathrm{TN}$ & $\mathrm{TN}$ & $\mathrm{TN}$ \\
\hline Langley Sth. & Drift & FN & FN & FN & FN & $\mathrm{TP}$ & $\mathrm{TP}$ & $\mathrm{TP}$ & $\mathrm{TP}$ & FN & $\mathrm{TP}$ & $\mathrm{TP}$ & $\mathrm{TP}$ \\
\hline Maple Ridge & Drift & $\mathrm{TP}$ & $\mathrm{TP}$ & $\mathrm{TP}$ & $\mathrm{TP}$ & $\mathrm{TP}$ & FN & $\mathrm{TP}$ & $\mathrm{TP}$ & FN & $\mathrm{TP}$ & $\mathrm{TP}$ & $\mathrm{TP}$ \\
\hline Nth. Delta & Drift & $\mathrm{TP}$ & $\mathrm{TP}$ & $\mathrm{TP}$ & $\mathrm{TP}$ & $\mathrm{TP}$ & $\mathrm{TP}$ & TP & $\mathrm{TP}$ & FN & $\mathrm{TP}$ & $\mathrm{TP}$ & $\mathrm{TP}$ \\
\hline Pitt Meadows & Drift & $\mathrm{TP}$ & $\mathrm{TP}$ & $\mathrm{TP}$ & $\mathrm{TP}$ & FN & $\mathrm{TP}$ & $\mathrm{TP}$ & $\mathrm{TP}$ & $\mathrm{FN}$ & $\mathrm{TP}$ & $\mathrm{TP}$ & $\mathrm{TP}$ \\
\hline Port Moody & Drift & $\mathrm{TP}$ & $\mathrm{TP}$ & $\mathrm{TP}$ & $\mathrm{TP}$ & TP & TP & $\mathrm{TP}$ & $\mathrm{TP}$ & TP & $\mathrm{TP}$ & $\mathrm{TP}$ & TP \\
\hline Richmond & Drift & FN & FN & FN & FN & FN & FN & FN & FN & FN & $\mathrm{TP}$ & $\mathrm{TP}$ & $\mathrm{TP}$ \\
\hline $\begin{array}{l}\text { Second } \\
\text { Narrows }\end{array}$ & OK & $\mathrm{TN}$ & $\mathrm{TN}$ & $\mathrm{TN}$ & FP & $\mathrm{TN}$ & $\mathrm{TN}$ & $\mathrm{TN}$ & $\mathrm{TN}$ & $\mathrm{TN}$ & FP & FP & FP \\
\hline Surrey East & OK & $\mathrm{TN}$ & $\mathrm{TN}$ & $\mathrm{TN}$ & $\mathrm{TN}$ & $\mathrm{TN}$ & $\mathrm{TN}$ & $\mathrm{TN}$ & $\mathrm{TN}$ & $\mathrm{TN}$ & $\mathrm{TN}$ & TN & $\mathrm{TN}$ \\
\hline \multicolumn{2}{|c|}{ \# True States / Total Sites } & 0.78 & 0.78 & 0.78 & 0.67 & 0.78 & 0.78 & 0.89 & 0.89 & 0.44 & 0.89 & 0.89 & 0.89 \\
\hline
\end{tabular}

From this, either $t_{d}=72$ or $t_{d}=120$ would be suitable. 
Table S2: Sensitivity analysis for the selection of time index, $t_{f}$, using comparisons on Type 1 (False Positive, FP) \& 2 (False Negative, FN) errors based on the co-located GSS instrument data and single sensor assessment (SSA) states for $t_{d}=72$ and $t_{d}=120$. (TP: true positive; TN: true negative)

\begin{tabular}{|c|c|c|c|c|c|c|c|c|c|c|c|c|c|}
\hline \multirow[t]{2}{*}{ Site $\left(t_{d}=72\right)$} & \multirow[t]{2}{*}{ SSA } & \multicolumn{4}{|l|}{$\hat{\mathbf{a}}_{0}$} & \multicolumn{4}{|l|}{$\hat{\mathbf{a}}_{1}$} & \multicolumn{4}{|l|}{$P_{K S}$} \\
\hline & & $\begin{array}{l}t_{f}= \\
24\end{array}$ & $\begin{array}{l}t_{f}= \\
72\end{array}$ & $\begin{array}{l}t_{f}= \\
120\end{array}$ & $\begin{array}{l}t_{f}= \\
168\end{array}$ & $\begin{array}{l}\mathrm{t}_{\mathrm{f}}= \\
24\end{array}$ & $\begin{array}{l}t_{f}= \\
72\end{array}$ & $\begin{array}{l}t_{f}= \\
120\end{array}$ & $\begin{array}{l}t_{f}= \\
168\end{array}$ & $\begin{array}{l}t_{f}= \\
24\end{array}$ & $\begin{array}{l}t_{\mathrm{f}}= \\
72\end{array}$ & $\begin{array}{l}t_{f}= \\
120\end{array}$ & $\begin{array}{l}t_{f}= \\
168\end{array}$ \\
\hline Abbotsford & $\mathrm{OK}$ & FP & FP & $\mathrm{TN}$ & $\mathrm{TN}$ & $\mathrm{TN}$ & $\mathrm{TN}$ & $\mathrm{TN}$ & $\mathrm{TN}$ & FP & $\mathrm{TN}$ & $\mathrm{TN}$ & $\mathrm{TN}$ \\
\hline Langley Sth. & Drift & $\mathrm{TP}$ & FN & FN & FN & $\mathrm{TP}$ & $\mathrm{TP}$ & $\mathrm{TP}$ & $\mathrm{TP}$ & $\mathrm{TP}$ & $\mathrm{TP}$ & $\mathrm{TP}$ & $\mathrm{TP}$ \\
\hline Maple Ridge & Drift & $\mathrm{TP}$ & $\mathrm{TP}$ & $\mathrm{TP}$ & $\mathrm{TP}$ & $\mathrm{TP}$ & $\mathrm{TP}$ & $\mathrm{TP}$ & $\mathrm{TP}$ & $\mathrm{TP}$ & $\mathrm{TP}$ & $\mathrm{TP}$ & $\mathrm{TP}$ \\
\hline Nth. Delta & Drift & $\mathrm{TP}$ & $\mathrm{TP}$ & $\mathrm{TP}$ & $\mathrm{TP}$ & $\mathrm{TP}$ & $\mathrm{TP}$ & $\mathrm{TP}$ & $\mathrm{TP}$ & $\mathrm{TP}$ & $\mathrm{TP}$ & $\mathrm{TP}$ & $\mathrm{TP}$ \\
\hline Pitt Meadows & Drift & TP & $\mathrm{TP}$ & TP & $\mathrm{TP}$ & TP & $\mathrm{TP}$ & TP & $\mathrm{TP}$ & TP & $\mathrm{TP}$ & TP & $\mathrm{TP}$ \\
\hline Port Moody & Drift & $\mathrm{TP}$ & $\mathrm{TP}$ & $\mathrm{TP}$ & $\mathrm{TP}$ & $\mathrm{TP}$ & $\mathrm{TP}$ & $\mathrm{TP}$ & $\mathrm{TP}$ & $\mathrm{TP}$ & $\mathrm{TP}$ & $\mathrm{TP}$ & $\mathrm{TP}$ \\
\hline Richmond & Drift & $\mathrm{TP}$ & $\mathrm{TP}$ & FN & FN & FN & FN & FN & FN & $\mathrm{TP}$ & $\mathrm{TP}$ & $\mathrm{TP}$ & $\mathrm{TP}$ \\
\hline $\begin{array}{l}\text { Second } \\
\text { Narrows }\end{array}$ & $\mathrm{OK}$ & $\mathrm{FN}$ & FP & $\mathrm{TN}$ & $\mathrm{TN}$ & $\mathrm{TN}$ & $\mathrm{TN}$ & $\mathrm{TN}$ & $\mathrm{TN}$ & FP & FP & FP & FP \\
\hline Surrey East & $\mathrm{OK}$ & $\mathrm{TN}$ & $\mathrm{TN}$ & $\mathrm{TN}$ & $\mathrm{TN}$ & $\mathrm{TN}$ & $\mathrm{TN}$ & $\mathrm{TN}$ & $\mathrm{TN}$ & $\mathrm{TN}$ & $\mathrm{TN}$ & $\mathrm{TN}$ & $\mathrm{TN}$ \\
\hline \multicolumn{2}{|c|}{ \# True States / Total Sites } & 0.78 & 0.78 & 0.78 & 0.78 & 0.89 & 0.89 & 0.89 & 0.89 & 0.78 & 0.89 & 0.89 & 0.89 \\
\hline
\end{tabular}

\begin{tabular}{|c|c|c|c|c|c|c|c|c|c|c|c|c|c|}
\hline \multirow[t]{2}{*}{ Site $\left(t_{d}=120\right)$} & \multirow[t]{2}{*}{ SSV } & \multicolumn{4}{|l|}{$\hat{\mathbf{a}}_{0}$} & \multicolumn{4}{|l|}{$\hat{\mathbf{a}}_{1}$} & \multicolumn{4}{|l|}{$P_{K S}$} \\
\hline & & $\begin{array}{l}t_{f}= \\
24\end{array}$ & $\begin{array}{l}t_{f}= \\
72\end{array}$ & $\begin{array}{l}t_{f}= \\
120\end{array}$ & $\begin{array}{l}t_{f}= \\
168\end{array}$ & $\begin{array}{l}\mathrm{t}_{\mathrm{f}}= \\
24\end{array}$ & $\begin{array}{l}t_{f}= \\
72\end{array}$ & $\begin{array}{l}t_{f}= \\
120\end{array}$ & $\begin{array}{l}t_{\mathrm{f}}= \\
168\end{array}$ & $\begin{array}{l}\mathrm{t}_{\mathrm{f}}= \\
24\end{array}$ & $\begin{array}{l}t_{f}= \\
72\end{array}$ & $\begin{array}{l}t_{f}= \\
120\end{array}$ & $\begin{array}{l}t_{\mathrm{f}}= \\
168\end{array}$ \\
\hline Abbotsford & $\mathrm{OK}$ & FP & FP & $\mathrm{TN}$ & $\mathrm{TN}$ & TN & $\mathrm{TN}$ & $\mathrm{TN}$ & $\mathrm{TN}$ & FP & FP & $\mathrm{TN}$ & $\mathrm{TN}$ \\
\hline Langley Sth. & Drift & $\mathrm{FN}$ & FN & FN & FN & $\mathrm{TP}$ & $\mathrm{TP}$ & $\mathrm{TP}$ & $\mathrm{TP}$ & $\mathrm{TP}$ & $\mathrm{TP}$ & $\mathrm{TP}$ & $\mathrm{TP}$ \\
\hline Maple Ridge & Drift & TP & $\mathrm{TP}$ & $\mathrm{TP}$ & $\mathrm{TP}$ & TP & TP & $\mathrm{TP}$ & TP & $\mathrm{TP}$ & $\mathrm{TP}$ & TP & $\mathrm{TP}$ \\
\hline Nth. Delta & Drift & $\mathrm{TP}$ & $\mathrm{TP}$ & $\mathrm{TP}$ & $\mathrm{TP}$ & $\mathrm{TP}$ & $\mathrm{TP}$ & $\mathrm{TP}$ & $\mathrm{TP}$ & $\mathrm{TP}$ & $\mathrm{TP}$ & $\mathrm{TP}$ & $\mathrm{TP}$ \\
\hline Pitt Meadows & Drift & $\mathrm{TP}$ & $\mathrm{TP}$ & $\mathrm{TP}$ & $\mathrm{TP}$ & $\mathrm{TP}$ & $\mathrm{TP}$ & $\mathrm{TP}$ & $\mathrm{TP}$ & $\mathrm{TP}$ & $\mathrm{TP}$ & $\mathrm{TP}$ & $\mathrm{TP}$ \\
\hline Port Moody & Drift & TP & TP & TP & TP & TP & TP & TP & TP & TP & TP & TP & $\mathrm{TP}$ \\
\hline Richmond & Drift & $\mathrm{TP}$ & FN & FN & FN & FN & FN & FN & FN & $\mathrm{TP}$ & $\mathrm{TP}$ & $\mathrm{TP}$ & $\mathrm{TP}$ \\
\hline $\begin{array}{l}\text { Second } \\
\text { Narrows }\end{array}$ & $\mathrm{OK}$ & FP & FP & $\mathrm{TN}$ & $\mathrm{TN}$ & $\mathrm{TN}$ & $\mathrm{TN}$ & $\mathrm{TN}$ & $\mathrm{TN}$ & FP & FP & FP & FP \\
\hline Surrey East & $\mathrm{OK}$ & $\mathrm{TN}$ & $\mathrm{TN}$ & $\mathrm{TN}$ & $\mathrm{TN}$ & $\mathrm{TN}$ & $\mathrm{TN}$ & $\mathrm{TN}$ & $\mathrm{TN}$ & $\mathrm{TN}$ & $\mathrm{TN}$ & $\mathrm{TN}$ & $\mathrm{TN}$ \\
\hline \multicolumn{2}{|c|}{ \# True States / Total Sites } & 0.67 & 0.56 & 0.78 & 0.78 & 0.89 & 0.89 & 0.89 & 0.89 & 0.78 & 0.78 & 0.89 & 0.89 \\
\hline
\end{tabular}

From this, either $t_{f}=120$ or $t_{f}=168$ would be suitable. It was found for both sensitivity analyses that the data worked best for longer time indices (as expected). Due to this, the shortest suitable time index was selected in order to achieve swifter detection times, and so $t_{d}$ $=72$ and $t_{f}=120$ were selected. 
Table S3: Time of drift using the three verification techniques for the 18 residential locations using the similar land-use and network median proxy. Total is the sum of failures over this period for each technique. (SSA - Single Sensor Assessment).

\begin{tabular}{|c|c|c|c|c|c|c|c|}
\hline \multirow[t]{2}{*}{ Location } & \multirow[t]{2}{*}{ SSA } & \multicolumn{3}{|c|}{ Similar land-use proxy } & \multicolumn{3}{|c|}{ Network proxy } \\
\hline & & $\hat{a}_{0}$ & $\hat{a}_{1}$ & $p_{K S}$ & $\hat{a}_{0}$ & $\hat{a}_{1}$ & $p_{K S}$ \\
\hline Abbotsford & NA & NA & NA & NA & NA & NA & NA \\
\hline Aldergrove & $16 / 7$ & $16 / 7$ & $07 / 7$ & $08 / 7$ & $16 / 7$ & $18 / 7$ & $27 / 7$ \\
\hline Burnaby & $01 / 7$ & $01 / 7$ & $01 / 7$ & NA & $01 / 7$ & $01 / 7$ & $07 / 7$ \\
\hline Capitol Hill & $13 / 7$ & $01 / 7$ & $01 / 7$ & $01 / 7$ & $01 / 7$ & $01 / 7$ & NA \\
\hline Hayward & $22 / 7$ & $16 / 7$ & NA & NA & $17 / 7$ & NA & $22 / 7$ \\
\hline Horseshoe & $09 / 7$ & $12 / 7$ & $11 / 7$ & NA & $12 / 7$ & $12 / 7$ & NA \\
\hline Langley $\mathrm{L}$. & NA & $01 / 7$ & $02 / 7$ & NA & $01 / 7$ & $01 / 7$ & NA \\
\hline Langley & $13 / 7$ & $08 / 7$ & NA & $09 / 7$ & $15 / 7$ & NA & $15 / 7$ \\
\hline Maple Ridge & $16 / 7$ & $09 / 7$ & $01 / 7$ & $06 / 7$ & NA & $13 / 7$ & $20 / 7$ \\
\hline Mission & NA & NA & NA & NA & $26 / 7$ & NA & $23 / 7$ \\
\hline North Delta & $09 / 7$ & $04 / 7$ & $01 / 7$ & $01 / 7$ & $04 / 7$ & $04 / 7$ & $02 / 7$ \\
\hline North Tsawwassen & $15 / 7$ & $187 /$ & $18 / 7$ & $18 / 7$ & $23 / 7$ & $18 / 7$ & $18 / 7$ \\
\hline Renfrew & $09 / 7$ & NA & $16 / 7$ & $17 / 7$ & $16 / 7$ & $05 / 7$ & $20 / 7$ \\
\hline Richmond & $26 / 7$ & NA & NA & NA & NA & $06 / 7$ & NA \\
\hline Sou & NA & $01 / 7$ & $01 / 7$ & NA & $01 / 7$ & $01 / 7$ & NA \\
\hline Surrey & NA & NA & NA & NA & $23 / 7$ & NA & NA \\
\hline Thirty-Second & NA & $08 / 7$ & $26 / 7$ & $01 / 7$ & NA & NA & NA \\
\hline White Rock & NA & $01 / 7$ & $01 / 7$ & NA & $01 / 7$ & $01 / 7$ & $\mathrm{NA}$ \\
\hline Total & 11 & 13 & 12 & 8 & 14 & 12 & 8 \\
\hline
\end{tabular}

Further analysis of this dataset and details of GSS instruments and their use in the atmosphere have been published in:

- Bart, M., Williams, D. E., Ainslie, B., McKendry, I., Salmond, J., Grange, S. K., Henshaw, G. S. (2014). High density ozone monitoring using gas sensitive semiconductor sensors in the Lower Fraser Valley, British Columbia. Environmental Science and Technology, 48(7), 3970-3977

- Williams, D. E., Henshaw, G. S., Bart, M., Laing, G., Wagner, J., Naisbitt, S., \& Salmond, J. A. (2013). Validation of low-cost ozone measurement instruments suitable for use in an air-quality monitoring network. Measurement Science and Technology, 24, 065803

- Williams, D. E., Henshaw, G., Wells, B., Ding, G., Wagner, J., Wright, B., Yung, Y.F., Salmond, J. (2009). Development of Low-Cost Ozone Measurement Instruments Suitable for Use in an Air Quality Monitoring Network. Chemistry in New Zealand 73(1), 27-33

- Williams, D.E., Salmond, J., Yung, Y.F. Akaji, J., Wright, B., Wilson, J., Henshaw, G., Wells, D., Ding, G., Wagner, J., Laing, G. (2009) Development Of Low-Cost Ozone and Nitrogen Dioxide Measurement Instruments Suitable For Use In An Air Quality Monitoring Network, $8^{\text {th }}$ IEEE Conference on Sensors, Christchurch, NZ, IEEE Sensors, 1-3: $1099-1104$ 